\title{
SHOE-INTEGRATED SENSOR SYSTEM FOR WIRELESS GAIT ANALYSIS AND REAL-TIME FEEDBACK
}

\author{
Stacy J. Morris ${ }^{1}$, Joseph A. Paradiso ${ }^{2}$ \\ ${ }^{1}$ Harvard/MIT Division of Health Science and Technology: Medical Engineering, Cambridge, MA, USA \\ ${ }^{2}$ MIT Media Laboratory, Cambridge, MA, USA
}

\begin{abstract}
We are developing a sensor system for use in clinical gait analysis. This research involves the development of an on-shoe device that can be used for continuous and real-time monitoring of gait. This paper presents the design of an instrumented insole and a removable instrumented shoe attachment. Transmission of the data is in real-time and wireless, providing information about the three-dimensional motion, position, and pressure distribution of the foot. Using pattern recognition and numerical analysis of the calibrated sensor outputs, algorithms will be developed to analyze the data in real-time. Results will be validated by comparison to results from a commerical optical gait analysis system at the Massachusetts General Hospital (MGH) Biomoti on Lab.
\end{abstract}

Keywords - Gait analysis, sensors, wireless, real-time feedback

\section{INTRODUCTION}

At the present time, gait analysis is primarily carried out in one of two ways: in a motion laboratory, with full analysis of the motion of all body segments using highly accurate optical systems, or in a doctor's office with the physician making visual observations. The first method is expensive, requires the maintenance of a dedicated motion lab, and uses cumbersome equipment attached to the patient, but produces well-quantified and accurate results for short distances. The second method is inexpensive and does not require anything to be attached to the patient, but the results are qualitative and difficult to compare across multiple visits.

There is a need for a low cost device that falls in between these two methods, one that can provide quantitative and repeatable results. In addition, there is a need for monitoring gait over long periods of time; in particular, analysis of gait in Parkinson's patients would be greatly enhanced by studies looking at gait outside of the motion lab [1].

In the last decade, a number of systems capable of investigating a sub-group of gait characteristics have been developed. An insole capable of real-time pressure analysis at seven locations beneath each foot, but required the patient to be tethered via power and data lines to the computer [2]. Another insole based system has been able to discern an impressive amount of information about the temporal parameters of gait, such as changes in stride timing; this system is non-tethered, but does not provide real-time analysis [3]. Recently, a system with an instrumented insole and gyroscope mounted on the shoe has achieved good results in classifying the gait into four different phases, but does not appear to be capable of real-time analysis yet [4].

The precursor of this research resulted in an instrumented insole and shoe with a total of sixteen outputs per shoe, which were provided wirelessly and in real-time; however this device was not optimized for analysis of the sensor outputs, instead the shoe was worn by dancers who used the movement of their feet to control music [5].

These and many other systems have been able to provide detailed information about some aspect of the motion. Our research strives to be able to completely characterize the motion of the foot. In addition, we will provide the data in real-time, so that it can ultimately be used to give real-time feedback to the user.

\section{MethodolOGY}

\section{A. Hardware Development}

We have worked with our colleagues at the Massachusetts General Hospital (MGH) Biomotion Laboratory to determine the important requirements for our device, the ShoeIntegrated Gait Sensors (SIGS). The top-level functional requirements for the design of the SIGS are:

1. Attach removably to the subject's own walking shoes.

2. Effect no change in gait.

3. Characterize the motion of both feet.

4. Communicate and transmit the data wirelessly.

5. Provide validated kinematic data $\&$ analysis in realtime. The schematic of the SIGS is shown in Fig.1, and photos of the initial prototype are shown in Fig. 2. The insole (Fig.2, right) contains four FSRs (force sensitive resistors), two PVDFs (polyvinlylidine fluoride, a piezoelectric material), and two pairs of resistive bend sensors placed back to back to provide bi-directional bend. Two FSRs are located beneath the heel (medially and laterally), one FSR is located beneath the first metatarsal head, and one beneath the fourth and fifth metatarsal head, providing a coarse measurement of the pressure distribution beneath the foot. One PVDF is

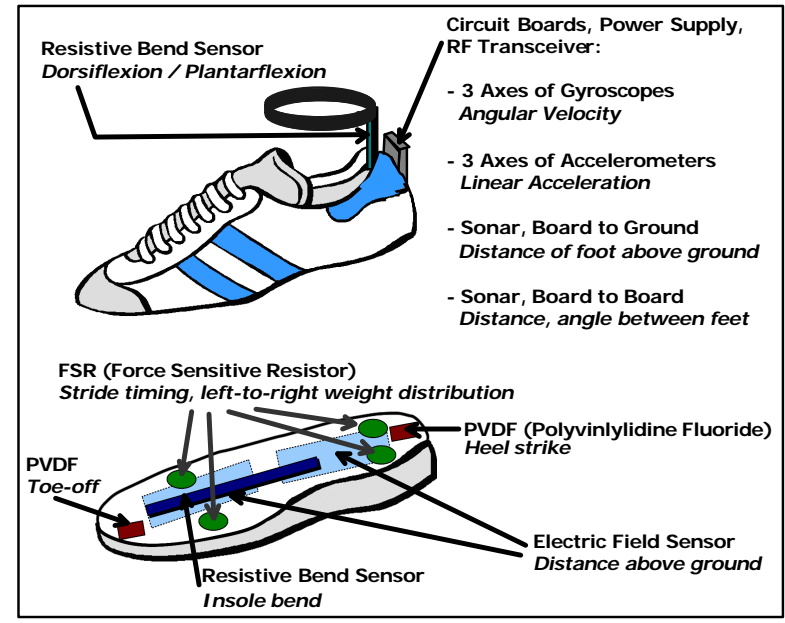

Figure 1. Schematic of the Shoe-Integrated Gait Sensors (SIGS) 


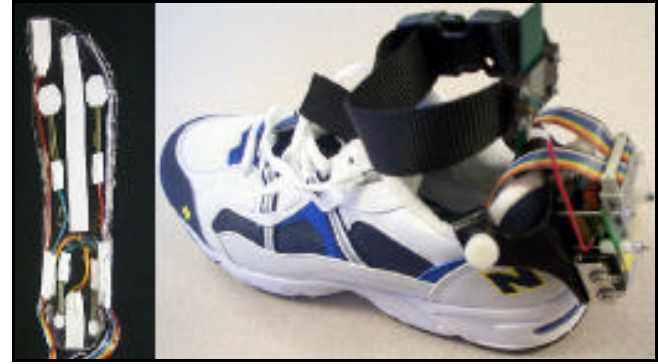

Figure 2. Photos of the first prototype of the SIGS

located beneath the heel, and the other is beneath the hallux (big toe), providing dynamic information about "heel strike" and "toe off." One pair of bend sensors is located at the back of the shoe and is inserted into an ankle strap; it bends about the back of the shoe and provides information about the extent of plantarflexion or dorsiflexion. The other pair is located in the insole to provide information about the extent of "roll" off the ball of the foot during walking.

The shoe attachment (Fig. 2, right) contains the circuit boards in a stacked configuration and the power supply (9V); the mass of the current prototype is approximately $200 \mathrm{~g}$. There is a circuit board for the insole sensors, another with a microcontroller and wireless transceiver, and a third with three axes each of gyroscopes and accelerometers; the redundancy in measurements will be used to reduce errors. A fourth board in final development will provide capacitive sensing and sonar to measure the distance to the ground, and sonar for measuring the distance and angle between shoes.

\section{B. Signal Analysis}

Initial results obtained with the prototype system are shown in Fig. 3. Current work is focused on the development of methods to analyze the data, including calibration and analysis of the outputs of the sensors, as well pattern recognition methods. Fine tuning of the sensors and corresponding circuits is ongoing (i.e. to prevent the PVDF signals from saturating).

\section{DISCUSSION AND CONCLUSION}

There is a clear need for a device which can provide quantitative analysis of gait in conditions outside of the motion lab. While this work is continuing to be developed, it shows great promise as a future contributor to clinical gait analysis. The initial results show significant breadth in measurement capability. We are currently working on analysis methods for this data; once these methods are implemented and validated with the commercial equipment at the MGH Biomotion lab, we will use our device to look at gait in different environments and at differences between the gait of various subjects.

\section{ACKNOWLEDGMENTS}

The authors would like to acknowledge their colleagues at the MIT Media Laboratory and the MGH Biomotion Lab for their work on this project, as well as the Whitaker Foundation and the Center for Integration of Medicine and Innovative Technology (CIMIT) for funding and support.

\section{REFERENCES}

[1] ME Morris, F Huxham, J McGinley, K Dodd, and R Iansek, "The biomechanics and motor control of gait in Parkinson disease," Clin Biomech, $2001 \mathrm{Jul} ; 16(6)$ : 459-70.

[2] HS Zhu HS, N Maalej, JG Webster, WJ Tompkins, P Bach-Y-Rita, and JJ Wertsch, "An umbilical data-acquisition system for measuring pressures between the foot and shoe," IEEE Trans Biomed Eng, 1990 Sep; 37(9): 908-11

[3] JM Hausdorff, Z Ladin, and JY Wei, "Footswitch system for measurement of the temporal parameters of gait," J Biomech, 1995 Mar; 28(3): 347-51.

[4] IP Pappas, MR Popovic, T Keller, V Dietz; and M Morari, "A reliable gait phase detection system," IEEE Trans Neural Syst Rehabil Eng, 2001 Jun; 9(2): 113-25.

[5] JA Paradiso, K Hsiao, AY Benbasat, and Z Teegarden, "Design and implementation of expressive footware," IBM systems journal, 2000; 39 (3): 511-519.

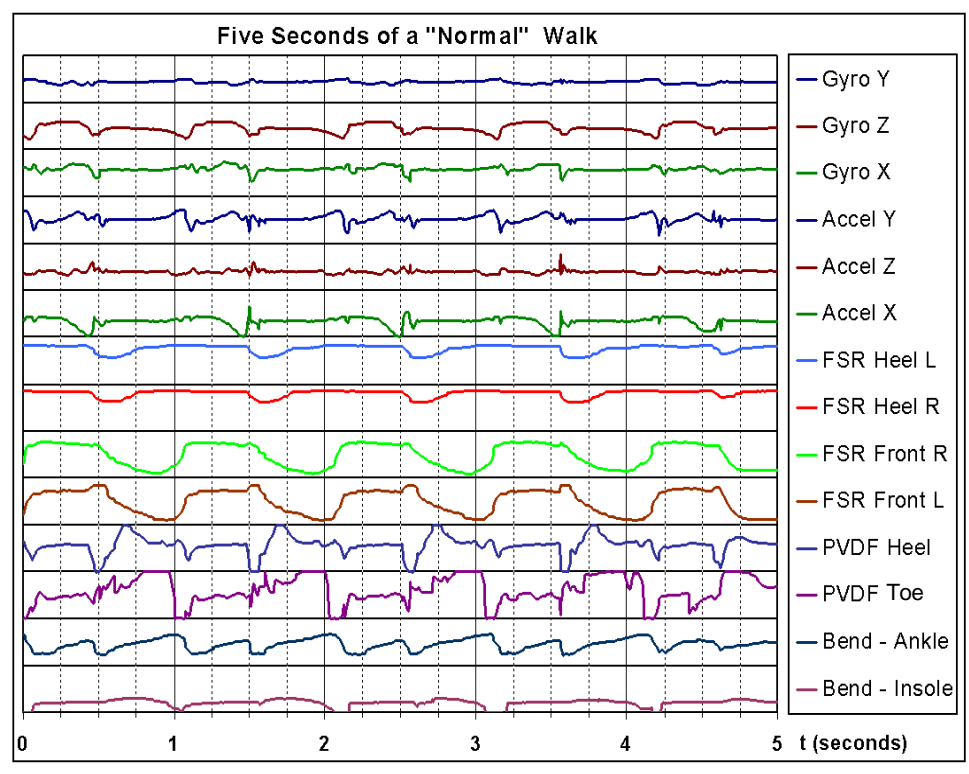

Figure 3. Initial results obtained with the SIGS

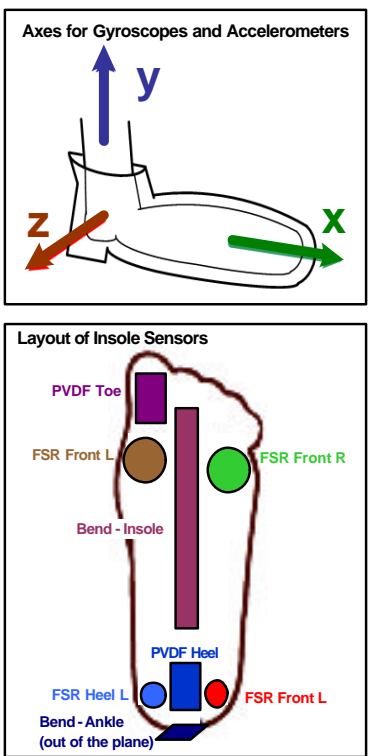

